\title{
PROTOCOLO DE ADAPTACIÓN DE ALEVINOS DE PAICHE Arapaima gigas AL CONSUMO DE ALIMENTO ARTIFICIAL EN CAUTIVERIO
}

\author{
Javier Velásquez ${ }^{1}$, Magali Del Risco ${ }^{1}$, Fred Chu-Koo ${ }^{2 *}$, Fernando Alcántara Bocanegra ${ }^{2}$, Carlos Chávez \\ Veintemilla', Palmira Padilla', Hugo Marichín Ayambo y Salvador Tello Martin ${ }^{2}$
}

\section{RESUMEN}

El paiche, Arapaima gigas, es un pez amazónico de régimen carnívoro y que en su medio natural se alimenta de peces y otros animales acuáticos. Una de las principales limitaciones que se observa en el manejo de paiche en cautiverio es la alimentación de los alevinos debido a que ellos solo aceptan peces forraje lo que eleva los costos de producción. El objetivo del trabajo fue establecer un protocolo eficaz de adaptación de los alevinos de paiche al consumo de alimento balanceado (peletizado o extrusado), procedimiento que será de mucha ayuda para los piscicultores, empresarios acuaristas y la comunidad académica de la región amazónica interesados en la cría, comercialización e investigación del paiche. En el presente estudio, un total de 48 alevinos de paiche que solo aceptaban alimento vivo (peces) fueron adiestrados exitosamente al consumo de alimento inerte (ración artificial balanceada) en un periodo de 23 días. El protocolo consta de 12 pasos y es usado en el Instituto de Investigaciones de la Amazonía Peruana (IIAP). Este procedimiento se basa en el suministro de carne de pescado fresco conjuntamente con alimento balanceado, realizando reducciones graduales en la proporción de carne y un aumento progresivo del alimento balanceado hasta que los alevinos de paiche aceptan la ración balanceada al 100\%. El periodo de adaptación y las proporciones de mezcla de alimentos pueden variar y ser particulares para cada nueva experiencia de adaptación pues estos dependerán de la calidad de agua y la edad o grado de desarrollo de los peces.

PALABRAS CLAVE: Paiche, Arapaima gigas, alevinos, protocolo, destete, alimento artificial.

\section{PROTOCOL FOR ADAPTING PAICHE Arapaima gigas FINGERLINGS TO FEED ON ARTIFICIAL DIETS IN CAPTIVITY}

\begin{abstract}
The paiche, Arapaima gigas, is a carnivore Amazonian fish which in the wild feeds on fish and other aquatic animals. Feeding their fingerlings is one of the main limitations observed when raising paiche in captivity since they only accept live fish which elevates the production costs. The objective of the present study was to provide an effective protocol for adapting paiche fingerlings to the consumption of artificial balanced foodstuff (pelleted or extruded diets) oriented to fish farmers, private entrepreneurs, aquarists and academic community of the Amazon region interested in raising, trading or researching paiche. A total of 48 fingerlings were trained successfully to the consumption of an artificial balanced foodstuff in a period of 23 days. The current protocol is based on 12 steps and it is used in the Peruvian Amazon Research Institute (IIAP). This procedure can also be denominated as a "progressive weaning", which is based on giving balanced food jointly with meat of fresh fish, carrying out gradual reductions in the meat proportion and a progressive increase of the balanced food until the fingerlings do accept the artificial foodstuff to $100 \%$. The period of adaptation and the proportions of mixture of foods could vary and may be particular for each new experience of adaptation, so that it will also depends on environmental factors such as, water quality and the age or developmental degree of the fingerlings.
\end{abstract}

KEYWORDS: Paiche, Arapaima gigas, fingerlings, protocol, artificial foodstuff.

1 Universidad Nacional de la Amazonía Peruana (UNAP). Tesista del Programa de Ecosistemas Acuáticos del IIAP. Iquitos, Perú. e-mail: javiervelasquez@hotmail.com

2 Instituto de Investigaciones de la Amazonía Peruana (IIAP). Programa de Ecosistemas Acuáticos (PEA). Centro de Investigaciones de Quistococha (CIO). Carretera lquitos - Nauta, Km. 4.5, Iquitos, Perú. e-mail: fchuk20@yahoo.com

* Autor para correspondencia. Dirección: Urbanización Juan Pablo II, Módulo 50A, Dpto. 101. San Juan Bautista, Iquitos. E-mail: fchuk20@yahoo.com. Celular: (065) 979-6120.RPM \#700038 


\section{INTRODUCCIÓN}

El paiche, Arapaima gigas, es una especie oriunda de la Amazonía peruana, sujeto a importantes estudios para su incorporación al cultivo como una forma de asegurar su uso sostenible y conservación debido a la alta presión de pesca que afecta seriamente a sus poblaciones en medio natural. Es uno de los peces de mayor tamaño en los cuerpos de agua dulce tropicales, alcanzando 2.5 metros de longitud total y hasta 250 kilos de peso. (Saint-Paul 1986; Rebaza et al. 1999; Padilla et al. 2003).

El paiche es un recurso pesquero de gran importancia económica, tanto por la calidad y cantidad de carne por individuo, como por las posibilidades de comercialización de los aionomorfos (larva y alevinos) producidos en ambientes controlados. La carne, además de ser deliciosa, prácticamente esta desprovista de espinas, su textura permite la elaboración de diversos platos para el consumo humano. El filete de paiche tiene alta aceptación en el mercado nacional e internacional por su calidad organoléptica (e.g. sabor y ausencia de espinas). El cuero del paiche que representa el $10 \%$ del peso del animal puede ser aprovechado en la industria, como materia prima para carteras, zapatos y cinturones, contribuyendo de manera importante al incremento económico del cultivo de esta especie (Imbiriba et al. 1996).

En los últimos años el paiche se ha perfilado como una especie de gran importancia para la piscicultura por su alto valor comercial en el mercado nacional e internacional en razón a la excelente calidad de su carne carente de espinas intramusculares (Imbiriba, 2001). En condiciones de cultivo, este pez puede superar los $12 \mathrm{~kg}$ de peso al primer año, con un crecimiento que casi triplica al del salmón y es incluso superior a la cobia que alcanza entre 7 a $10 \mathrm{~kg}$ en el mismo periodo (Chu-Koo et al. 2007).

En este orden, desde hace algunos años el Instituto de Investigaciones de la Amazonia Peruana (IIAP) viene desarrollando una serie de investigaciones enfocadas en aspectos de cultivo, nutrición, genética y reproducción impulsando, además, un programa de crianza de esta especie en estanques de productores de los departamentos de Loreto, San Martín, Ucayali y la provincia de Leoncio Prado (Huánuco) con el fin de incrementar la producción de alevinos para engorde y producción de filete y fundamentalmente, para reducir la presión de pesca sobre las poblaciones naturales.

A pesar de que normalmente las conversiones alimenticias de especies carnívoras son elevadas, la ganancia del peso diario del paiche es notable, lo que confirma su excelente potencial para el cultivo (Venturieri \& Bernardino, 1999). Sin embargo la crianza del paiche se dificulta por tratarse de un pez carnívoro que consecuentemente no acepta de manera voluntaria raciones balanceadas (Cavero et al. 2003). En tal sentido, para desarrollar un proceso de cultivo en base a una dieta artificial, es necesario tener un sistema de adaptación de los alevinos de paiche al consumo de dicho alimento; en el presente informe se describe la metodología empleada para lograr una aceptación total de alimento balanceado en 23 días de tratamiento.

El presente protocolo es solo uno de los productos del esfuerzo dedicado por el Centro de Investigaciones de Quistococha (CIQ), sede del Programa de Ecosistemas Acuáticos del IIAP orientados a mejorar el conocimiento existente sobre el manejo de los recursos piscícolas de la Amazonia peruana en condiciones controladas.

\section{MATERIALES Y MÉTODOS}

\section{ÁREADEESTUDIO}

El trabajo se realizó en las instalaciones del CIQ, ubicado en la comunidad de Quistococha, km. 4.5 de la carretera Iquitos-Nauta en la, provincia de Maynas, departamento de Loreto.

\section{MATERIALES YEQUIPOS}

- Tanques de cemento de 1.5 × 0.7 x $0.9 \mathrm{~m}$, revestidos con mayólicas con una profundidad (altura) de agua variable (entre 30 a $40 \mathrm{~cm}$ )

- 45 alevinos de paiche Arapaima gigas

- Peces forraje de las especies: mojarrita (Moenkhausia dichroura) o guppy (Poecilia reticulata)

- $10 \mathrm{~kg}$ de filete de doncella (Pseudoplatystoma fasciatum) o mota (Callophysus macropterus)

- $15 \mathrm{~kg}$ de alimento artificial para peces del tipo extrusado con $45 \%$ de proteína bruta formulada y elaborada en la Planta de Producción de Alimento Extrusado del IIAP.

- Molino de mano.

\section{PROCEDIMIENTO}

Antes de iniciar el estudio los 45 alevinos fueron criados y alimentados estrictamente con peces forraje de $2 \mathrm{~cm}$ de longitud en un solo tanque de cemento revestido de mayólicas. Al iniciarse el estudio, grupos de cinco peces de aproximadamente 80 gramos de peso fueron colocados en nueve tanques ( 45 peces en total) y fueron alimentados cada dos horas, desde las 8:00 a.m. hasta las 4:00 p.m. en raciones ad libitum (hasta la saciedad aparente).

Según lo recomendado por Person-Leruyet et al. (1993) se inició la alimentación artificial realizando el 
"destete progresivo", suministrando peces forraje de1 a $2 \mathrm{~cm}$ de longitud muertos para adaptar a los peces al consumo de alimento inerte.

Luego de la aceptación total del alimento inerte, en la siguiente etapa se les proporcionó pellets elaborados con carne molida de filete de bagres (porque no poseen espinas intramusculares que puedan dificultar el consumo por los alevinos). Al principio se notó poca aceptación del alimento pero en las horas subsiguientes, hubo aumento gradual de la oferta de la dieta. El proceso ha continuado hasta que observamos la aceptación total del alimento al segundo día de tratamiento.

El objetivo de la siguiente etapa fue el reconocimiento del alimento balanceado y la aceptación del sabor y textura por los alevinos. Para la prueba, la dieta fue elaborada con un $95 \%$ de carne molida de pescado y un $5 \%$ de polvo de alimento extrusado preparado en pellets y secado parcialmente para que los peces comiencen a reconocer la textura menos blanda y más seca. Los pellets fueron mayores al medio centímetro de diámetro para facilitar la ingestión del producto.

Luego de la aceptación total del pellet, fue reducida la pasta de pescado a un $90 \%$ y el aumento del polvo de alimento extrusado a un $10 \%$. Este producto final fue secado en mayor tiempo para obtener una consistencia más sólida y similar al alimento estrusado puro. Este tratamiento continuó hasta lograr la aceptación total del producto, para luego modificar nuevamente los porcentajes de los ingredientes aplicados en una nueva etapa.

Hasta el día 22 se elaboró raciones con $60 \%$ de polvo de alimento balanceado y $40 \%$ de pasta de pescado observándose buena aceptación. Finalmente el día 23 se proporcionó alimento extruído puro y entero observándose su aceptación total.

Los pasos simplificados para lograr la adaptación de los alevinos de paiche al alimento balanceado se muestran en la (Tabla 1).

Tabla 1. Resumen del proceso de adaptación de alevinos de paiche al alimento balanceado (peletizado o extrusado) realizado en el Centro de Investigaciones de Quistococha del IIAP, Loreto, Perú.

\begin{tabular}{|c|c|c|c|c|c|}
\hline \multicolumn{6}{|c|}{ ADAPTACIÓN DE ALEVINOS DE PAICHE } \\
\hline 1-3 día & 4-5 día & 6 día & 7-8 día & 9-10 día & 11-12 día \\
\hline $\begin{array}{l}\text { peces forraje } \\
\text { estrujados }\end{array}$ & $\begin{array}{l}\text { trocitos de filete } \\
\text { de pescado }\end{array}$ & $\begin{array}{c}95 \% \text { pasta pescado } \\
\text { y } 5 \% \text { polvo } \\
\text { de balanceado }\end{array}$ & $\begin{array}{c}90 \% \text { pasta pescado } \\
\text { y } 10 \% \text { polvo } \\
\text { de balanceado }\end{array}$ & $\begin{array}{c}85 \% \text { pasta pescado } \\
\text { y } 15 \% \text { polvo } \\
\text { de balanceado }\end{array}$ & $\begin{array}{c}80 \% \text { pasta pescado } \\
\text { y } 20 \% \text { polvo } \\
\text { de balanceado }\end{array}$ \\
\hline 13-14 día & 15-16 día & 17-18 día & 19-20 día & 21-22 día & 23 día \\
\hline $\begin{array}{c}75 \% \text { pasta pescado } \\
\text { y } 25 \% \text { polvo } \\
\text { de balanceado }\end{array}$ & $\begin{array}{c}65 \% \text { pasta pescado } \\
\text { y } 35 \% \text { polvo } \\
\text { de balanceado }\end{array}$ & $\begin{array}{c}60 \% \text { pasta pescado } \\
\text { y } 40 \% \text { polvo } \\
\text { de balanceado }\end{array}$ & $\begin{array}{l}50 \% \text { pasta pescado y } \\
50 \% \text { polvo de balanc. } \\
+ \text { alimento balanc. puro } \\
\text { en pequeñas cant. }\end{array}$ & $\begin{array}{l}40 \% \text { pasta pescado y } \\
60 \% \text { polvo de balanc. } \\
+ \text { alimento balanc. puro } \\
\text { en pequeñas can. }\end{array}$ & $\begin{array}{l}100 \% \text { alimento } \\
\text { balanceado puro }\end{array}$ \\
\hline
\end{tabular}

\section{RESULTADOS Y DISCUSIÓN}

\section{ADAPTACIÓN DE ALEVINOS DE PAICHE AL CONSUMO DE ALIMENTO ARTIFICIAL}

Según afirma Queiroz (1999), el paiche es una especie especializada en comer peces, de modo que no acepta de manera instantánea y voluntaria un alimento artificial siendo necesario desarrollar un proceso de adaptación al consumo del mismo. Sin embargo, Venturieri \& Bernardino (1999) y Crescêncio (2001) reportan que entrenar alevinos de paiche a consumir raciones secas es relativamente fácil en comparación con otras especies carnívoras.

Siguiendo a las recomendaciones de PersonLeruyet et al. (1993), fue constatada la efectividad del "destete progresivo" como estrategia para la adaptación al alimento artificial. En nuestra experiencia, utilizando porciones decrecientes de carne molida de pescado sin espinas mezclada con polvo de alimento artificial del tipo extrusado en proporciones crecientes, demostró una gran efectividad logrando la adaptación total de los peces al consumo de una dieta extrusada en alrededor de tres semanas. Este resultado guarda relación con García et al. (2002), quienes afirman que los periodos de adaptación a los alimentos balanceados en paiche generalmente comprenden de 7 a 30 días.

Por otro lado, Aldea (2002), consiguió la adaptación del paiche al alimento balanceado en dos meses de entrenamiento, aplicando un proceso gradual de suministro de pescado y alimento balanceado por separado. Nuestro protocolo combina ambos insumos haciendo que la adaptación sea más rápida y menos estresante para los peces. En el cultivo de estas especies una adaptación rápida al alimento balanceado es muy importante para poder obtener la mayor ganancia de peso en el menor tiempo posible. 


\section{RECOMENDACIONES}

Los resultados del protocolo aplicado en el presente trabajo pueden ser aplicados para desarrollar un proceso de adaptación rápida al consumo de alimento balanceado. Sin embargo el cronograma y las proporciones de los insumos, pescado y balanceado, pueden variar y ser particulares para cada nueva experiencia de adaptación porque los tiempos de aceptación dependerán además de los factores ambientales, del grado de desarrollo de los alevinos.

El proceso de adaptación debe ser ejecutado en jaulas o en corrales de precria instalados en el estanque definitivo, para evitar el estrés por traslados y variaciones en la calidad de agua.

Finalmente, es importante considerar que la adaptación al alimento artificial debe iniciarse cuando los alevinos alcanzan entre los 8 a $10 \mathrm{~cm}$ y nunca dejar que sobrepasen los $15 \mathrm{~cm}$ puesto que en tallas superiores a este limite el adiestramiento es más difícil de lograr.

\section{BIBLIOGRAFÍA}

ALDEA GM. Cultivo de paiche, Arapaima gigas (Cuvier, 1829) con dietas artificiales en jaulas flotantes. Tesis para optar el Título de Biólogo. Universidad Nacional de la Amazonía Peruana. Iquitos-Perú. 54 pp. 2002.

CAVERO BAS, PEREIRA-FILHO M, ROUBACH R, ITUASSU DR, GANDRA AL, CRESCÊNCIO R. Efeito da densidade de estocagem na homogeneidade do crescimento de juvenis de pirarucu em ambiente confinado. Pesq. Agropec. Bras., 2003; 38: 103-107.

CHU-KOO F, VALDIVIESO M, TELLO S, REBAZA M, REBAZA C, DEZA S, ALCÁNTARA F. Cría de paiche en jaulas flotantes. Análisis económico. Infopesca Internacional, 2007; 30 (abril - junio): 28-31.

CRESCÊNCIO R. Treinamento alimentar de alevinos de pirarucú, Arapaima gigas (Cuvier, 1829), utilizando atrativos alimentares. Disertação de Maestrado. Instituto Nacional de Pesquisas da Amazônia. Manaus-Brasil. 2001.
GARCÍA L, BARDALES F, MORI L. Influencia de tres tipos de dietas en el crecimiento de alevinos de paiche Arapaima gigas (CUVIER, 1870). I Congreso Iberoamericano Virtual de Acuicultura. 2002. [Consultado el 11 de noviembre del 2006] 10 pp. Disponible en: www.civa2002.org

IMBIRIBA E, LOURENÇO J, MOURA L, BRANDÃO L. Criação de pirarucú. Coleção Criar. Brasília, Brasil. No 264 pp. 1996.

IMBIRIBA EP. Potencial de criação do pirarucu Arapaima gigas em cativerio. Acta Amazonica, 2001;31(2):299-316.

PADILLA P, ALDEA M, ALCÁNTARA F. Adaptación del paiche, Arapaima gigas a la alimentación con dieta artificial. Informe Técnico PEA-IIAP. Iquitos-Perú. 22 pp. 2003.

PERSON-LERUYET J, ALEXANDRE J, THEBAUD L, MUGNIER C. Marine fish larvae feeding: Formulated diets or live prey? Journal of the World Aquaculture Society, 1993; 42:211-224.

QUEIROZ S. A preservacao e o uso sustentados dos pirarucus em Mamirahuá. Em: Estratégias para o manejo de recursos pesqueiros em Mamirahuá. 115 pp. 1999.

REBAZA M, ALCÁNTARA F, VALDIVIESO GM. Manual de Piscicultura del paiche Arapaima gigas. Edit. Manatí Gráfico S.A. Caracas Venezuela. 72 pp. 1999.

SAINT-PAUL U. Potential for aquaculture of South American fresh water fishes: a review. Aquaculture, 1986; 54(1):205-240.

VENTURIERI R, BERNARDINO G. Pirarucú, espécie ameaçada pode ser salva através do cultivo. Revista Panorama da Aqüicultura, 1999; 53:13-21. 